\title{
Long Lasting Efficiency of Monthly Vinblastine in a Case of Relapsed Anaplastic Large Cell Lymphoma
}

\author{
Ratha Mlis, Matthias Schell, Perrine Marec Bérard, Nathalie Bleyzac, Didier Frappaz
}

Institut d'Hématologie et d'Oncologie Pédiatrique, Lyon, France.

Email: mlis_ratha001@yahoo.com

Received February $28^{\text {th }}, 2013$; revised March $31^{\text {st }}, 2013$; accepted April $10^{\text {th }}, 2013$

Copyright (C) 2013 Ratha Mlis et al. This is an open access article distributed under the Creative Commons Attribution License, which permits unrestricted use, distribution, and reproduction in any medium, provided the original work is properly cited.

\begin{abstract}
Nearly $40 \%$ of children with Anaplastic Large cell lymphomas will relapse after a first-line strategy with short-pulse chemotherapy and reach a second remission in $30 \%$ to $60 \%$ with second line therapies including maintenance treatment with vinblastine or allogeneic hematopoietic stem-cell transplantation. The authors report a heavily pretreated case in second relapse who was maintained in third remission for 8 years with monthly vinblastine. He relapsed 16 weeks after discontinuation. This case demonstrates that monthly treatment with vinblastine may be sufficient to maintain a minimal disease. Oral compounds are now available and should be discussed in such situations.
\end{abstract}

Keywords: Anaplastic Large Cell Lymphoma; Vinblastine

\section{Introduction}

Spindle cell poisons are widely and empirically used in oncology and hematology though little is known about their pharmacokinetic/pharmacodynamic relationships. Weekly vinblastine at a dose of $6 \mathrm{mg} / \mathrm{m}^{2}$ had been reported as an efficient treatment for patients with recurrent anaplasic large cell lymphoma (ALCL) [1]. However, no consensus is available concerning frequency of injections and duration of treatments.

We report a case of ALCL with a persisting 3rd remission of 8 years provided by monthly vinblastine.

\section{Case Report}

A stage II CD 30+ ALCL skin lesion and frontal lymphadenopathy was diagnosed in 1992 in a 13-year-old male. He was treated by SFOP HM 89 protocol (Cyclophosphamide, Vincristine, Methotrexate and Adriamycin) followed by high dose chemotherapy by BEAM (BCNU, Etoposide, Aracytin and Melphalan, followed by bone marrow rescue).

First relapse occurred four years and three months after transplant. Fifty-two courses of weekly vinblastine at a dose of $6 \mathrm{mg} / \mathrm{m}^{2}$ provided a second prolonged complete remission. But a second relapse presented three months after treatment discontinuation. Intravenous vinblastine was restarted with progressive frequency decrease that sustained persisting remission: weekly for 6 months, every 15 days for 3 months, every 3 weeks for 5 years and finally monthly for 2 years and 6 months with good tolerance. It was delivered at home, through a permanent implantable device. Due to chemotherapy extravasation and uncertainty on the utility of such maintenance chemotherapy, the treatment was discontinued. 16 weeks after discontinuation, he relapsed marked by right leg lesion with inguinal lymphadenopathy, and further chemotherapy could never obtain an other remission. The patient died in 2008, 15 years after the diagnosis, in third relapse of a chemoresistant tumor progression.

\section{Discussion}

Vinblastine is specifically toxic for cytoskeleton microfilaments and microtubule and hence for the mitotic spindle, which results in metaphase blockage and necrosis of tumor cells. At very low, non cytotoxic dose, Vinblastin has an anti-angiogenic activity [2] that may be used to treat a wide spectrum of angiogenic-dependant diseases. Radiolabeled VLB infused at a dose of 0.167 $\mathrm{mg} / \mathrm{kg}$ follows a three-compartment open model system. The last phase being $\mathrm{t}_{1 / 2}=1173.0+/-65.0 \mathrm{~mm}$ [3], so that we can not expect significant vinblastine plasma levels beyond 7 days. Thus, a direct cytotoxic effect cannot explain that monthly injections of $6 \mathrm{mg} / \mathrm{m}^{2}$ were sufficient in our patient to maintain a minimal residual dis- 
ease for 8 years. Some alternative mechanisms are probably involved: antiangiogenic effect, restoration of anticancer immune response, restoration of tumor dormancy.

Brugières et al. reported that vinblastine as a single agent was an efficient treatment in patients with recurrent ALCL even when they had been previously treated with this drug: long-lasting remission was recorded in 8 of 13 patients treated for a first, second, third, or fifth relapse with weekly vinblastine at the same dose of $6 \mathrm{mg} / \mathrm{m}^{2}$ for 6 to 18 months [1]. This efficacy of retreatment with vinblastine has also been observed in low grade gliomas [4], and is rarely observed with other compounds. Our patient is unusual both because he had two successive responses to vinblastine, and because the last response was prolonged as long as this easily delivered treatment was not discontinued: i.e. 8 years. He could not be salvaged by classic chemotherapy. As oral compounds such as vinorelbine are now available, this raises the possibility of continuous treatment of such relapsing patients.

Thus, our case demonstrates that monthly injection may suffice in some ALCL patients to maintain minimal residual disease. These addicts to VLB could be treated by continuous oral vinorelbine until definitive treatments are available.

\section{REFERENCES}

[1] L. Brugieres, P. Quartier, M. C. Le Deley, H. Pacquement, Y. Perel, C. Bergeron, et al., "Relapses of Childhood Anaplastic Large-Cell Lymphoma: Treatment Results in a Series of 41 Children-A Report from the French Society of Pediatric Oncology," Annals of Oncology, Vol. 11, No. 1, 2000, pp. 53-58. doi:10.1023/A:1008352726155

[2] M. A. Jordan, D. Thrower and L. Wilson, "Effects of Vinblastine, Podophyllotoxin and Nocodazole on Mitotic Spindles. Implications for the Role of Microtubule Dynamics in Mitosis," Journal of Cell Science, Vol. 102, 1992, pp. 401-416.

[3] R. J. Owellen, C. A. Hartke and F. O. Hains, "Pharmacokinetics and Metabolism of Vinblastine in Humans," Cancer Research, Vol. 37, No. 8, 1977, pp. 2597-2602.

[4] E. Bouffet, R. Jakacki, S. Goldman, D. Hargrave, C. Hawkins, M. Shroff, et al., "Phase II Study of Weekly Vinblastine in Recurrent or Refractory Pediatric Low-Grade Glioma," Journal of Clinical Oncology, Vol. 30, No. 12, 2012, pp. 1358-1363. 